\title{
Universal Padé approximants and their behaviour on the boundary
}

\author{
Ilias Zadik
}

\begin{abstract}
There are several kinds of universal Taylor series. In one such kind the universal approximation is required at every boundary point of the domain of definition $\Omega$ of the universal function $f$. In another kind the universal approximation is not required at any point of $\partial \Omega$ but in this case the universal function $f$ can be taken smooth on $\bar{\Omega}$ and, moreover, it can be approximated by it's Taylor partial sums on every compact subset of $\bar{\Omega}$. Similar generic phenomena hold when the partial sums of the Taylor expansion of the universal function are replaced by some Padé approximants of it.

In the present paper we show that in the case of Padé approximants, if $\Omega$ is an open set and $S, T$ are two subsets of $\partial \Omega$ that satisfy some conditions, then there exists a universal function $f \in H(\Omega)$ which is smooth on $\Omega \cup S$ and has some Padé approximants that approximate $f$ on each compact subset of $\Omega \cup S$ and simultaneously obtain universal approximation on each compact subset of $(\mathbb{C} \backslash \bar{\Omega}) \cup$ $T$. A sufficient condition for the above to happen is $\bar{S} \cap \bar{T}=\emptyset$, while a necessary and sufficient condition is not known.
\end{abstract}

\section{Introduction}

Universality is a generic phenomenon of chaotic behavior of approximation [2]. One can say that a universal series of functions is a series of functions that are so extremely divergent that subsequences of them approximate lots of different functions on some sets.

A basic example of this phenomenon is the case of universal Taylor series, where the partial sums of the Taylor expansion of a holomorphic function $f$ approximate many functions on some compact sets outside the domain of definition $\Omega$ of $f$, with 
respect to the Euclidean distance. The history started with Fekete [15] before 1914, who introduced the first universal Taylor series on the real line. In the early 70's Luh [9] and independently Chui and Parnes [3] proved the existence of a Taylor series around zero with radius of convergence equal to 1 whose partial sums approximate uniformly any polynomial function on any compact set $K \subset \mathbb{C} \mid \bar{D}=\{z \in \mathbb{C}|| z \mid>1\}$ with connected complement. In [1], Nestoridis showed that there exists a universal Taylor series around zero with radius of convergence equal to 1 , where the approximation we described above is valid also on compact sets $K$ that can touch the boundary $\partial D=\{z \in \mathbb{C}|| z \mid=1\}$. Moreover, he proved in [12] that generically every function $f \in H(\Omega)$, where $\Omega$ is a simply connected domain, has universal Taylor series with respect to any center $\zeta \in \Omega$.

These series have universal approximating properties on every compact set $K$ in $\mathbb{C} \backslash \Omega$ with connected complement.

It is also proved in [7] that there exists a function $f \in H(\Omega)$ with the above properties which is also smooth on $\partial \Omega$, provided that the compact sets $K$ in $\mathbb{C} \backslash \Omega$ where the universal approximation takes place do not touch the boundary.

Recently, the partial sums of the Taylor expansion of a function, which are polynomials, have been replaced by a set of Padé approximants of $f$, which are rational functions. Therefore, they can take the value $\infty$ as well. In accordance with universal Taylor series, several results were proved in this new direction([4], [5], [13], [14]). In these new results it is shown that the Padé approximants can approximate any rational function uniformly with respect to the chordal metric on any compact set contained in $\mathbb{C} \backslash \bar{\Omega}[14$, or in $\mathbb{C} \backslash \Omega$ [13]. Moreover, it was proved in [6] that generically every function $f \in H(\Omega)$ can be approximated on any compact set in $\Omega$ by it's Padé approximants with respect to the Euclidean metric this time.

Thus, the above results assure us that generically, every function in $H(\Omega)$ can be approximated by some of it's Padé approximants and, simultaneously, the same Padé approximants realize universal Padé approximation of any rational function outside of $\bar{\Omega}$.

The question that naturally arises is what can be achieved on the boundary of $\Omega$. In [13, the universal approximation was extended to any compact subset of $\partial \Omega$ while in [14] the approximation of $f$ by its Padé approximants is valid not only on $\Omega$ but on $\partial \Omega$ as well. 
In the present paper we show that $\partial \Omega$ can be splitted in two disjoint parts $S$ and $T$, which must satisfy some conditions, so that the approximation towards $f$ is valid on compact subsets of $\Omega \cup S$ and the universal approximation of any rational function is valid on compact subsets of $(\mathbb{C} \backslash \bar{\Omega}) \cup T$. Our result is a general one and yields as corollaries all previous results. It is also generic in a closed subspace of $T^{\infty}\left(\Omega,\left(L_{n}\right)_{n \in \mathbb{N}}\right)$ where $L_{n}, n=1,2, \ldots$ are compact subsets of $\bar{\Omega}$ with some specific properties and $T^{\infty}\left(\Omega,\left(L_{n}\right)_{n \in \mathbb{N}}\right)$ is the set of all functions $f \in H(\Omega)$ where $\left.f^{(\ell)}\right|_{\left(L_{n} \cap \Omega\right)}$ is uniformly continuous for every $\ell \in \mathbb{N}_{0}, n \in \mathbb{N}$. The topology we consider on $T^{\infty}\left(\Omega,\left(L_{n}\right)_{n \in \mathbb{N}}\right)$ is endowed by the seminorms $\left\{\sup _{z \in\left(L_{n} \cap \Omega\right)}\left|f^{(\ell)}(z)\right| \mid \ell \in \mathbb{N}_{0}, n \in \mathbb{N}\right\}$ and therefore $T^{\infty}\left(\Omega_{n}\left(L_{n}\right)_{n \in \mathbb{N}}\right)$ becomes a Frechet space.

The closed subspace of $T^{\infty}(\Omega)=T^{\infty}\left(\Omega,\left(L_{n}\right)_{n \in \mathbb{N}}\right)$ where our result is generic, consists of the closure in $T^{\infty}\left(\Omega,\left(L_{n}\right)_{n \in \mathbb{N}}\right)$ of all rational functions with poles off $\bigcup_{n \in \mathbb{N}} L_{n}$.

Furthermore, we consider compact sets $K_{m} \subset \mathbb{C}, m=1,2, \ldots$, so that $K_{m} \cap L_{n}=\emptyset$ for all $m, n \in \mathbb{N}$ and we obtain universal Padé approximation on $K_{m}, m=1,2, \ldots$ with respect to the chordal metric. For special choices of the sequences $\left(L_{n}\right)_{n \in \mathbb{N}}$ and $\left(K_{m}\right)_{m \in \mathbb{N}}$ we cover all previously obtained generic results.

Finally, in the last section of the present paper we show that if the boundary $\partial \Omega$ of an open set $\Omega \subseteq \mathbb{C}$ contains two parts $S, T$ with $\bar{S} \cap \bar{T}=\emptyset$, then there always exists a function $f \in H(\Omega)$ such that some Padé approximants of $f$ approximate any rational function on the compact subsets of $(\mathbb{C} \backslash \bar{\Omega}) \cup T$ and simultaneously approximate $f$ on the compact subsets of $\Omega \cup S$, where $f$ is smooth.

\section{Preliminaries}

If we consider the one-point compactification $\mathbb{C} \cup\{\infty\}=\widetilde{\mathbb{C}}$ of $\mathbb{C}$, then a well known metric is the chordal metric $\chi$ on $\mathbb{C} \cup\{\infty\}$, where

$$
\chi(a, b)=\frac{|a-b|}{\sqrt{1+|a|^{2}} \sqrt{1+|b|^{2}}}, \text { for } a, b \in \mathbb{C}
$$

and $\chi(a, \infty)=\frac{1}{\sqrt{1+|a|^{2}}}$ for $a \in \mathbb{C}$, and $\chi(\infty, \infty)=0$;

Proposition 2.1. Let $K \subset \mathbb{C}$ be a compact set and $q=\frac{A}{B}$ a rational function, where the polynomials $A, B$ do not have a common zero in $\mathbb{C}$. Then there is a sequence $q_{j}=$ 
$\frac{A_{j}}{B_{j}}, j=1,2, \ldots$ where the polynomials $A_{j}$ and $B_{j}$ have coefficients in $\mathbb{Q}+i \mathbb{Q}$ and do not have any common zero in $\mathbb{C}$ for all $j$, such that $\sup _{z \in K} \chi\left(q_{j}(z), q(z)\right) \rightarrow 0$ as $j \rightarrow+\infty$.

The above proposition is well known. See [13].

Let $\zeta \in \mathbb{C}$ be fixed and

$$
f=\sum_{n=0}^{\infty} a_{n}(z-\zeta)^{n}
$$

be a formal power series $\left(a_{n}=a_{n}(f, \zeta)\right)$. This power series is often the Taylor development of a holomorphic function $f$ in a neighborhood of $\zeta$. Let $p$ and $q$ be two non negative integers. The Padé approximant $[f ; p / q]_{\zeta}(z)$ is defined to be a rational function $\phi$ regular at $\zeta$ whose Taylor development with center $\zeta$,

$$
\phi(z)=\sum_{n=0}^{\infty} b_{n}(z-\zeta)^{n},
$$

satisfies $b_{n}=a_{n}$ for all $0 \leq n \leq p+q$ and $\phi(z)=A(z) / B(z)$, where the polynomials $A$ and $B$ satisfy

$$
\operatorname{deg} A \leq p, \quad \operatorname{deg} B \leq q \text { and } B(\zeta) \neq 0 .
$$

It is not always true that such a rational function $\phi$ exists. And if it exists it is not always unique. For $q=0$, we always have such a unique $\phi$ which is

$$
[f ; p / q]_{\zeta}(z)=\sum_{n=0}^{p} a_{n}(z-\zeta)^{n} .
$$

For $q \geq 1$ the necessary and sufficient condition for existence and uniqueness is that the following $q \times q$ Hankel determinant is non-zero $([1])$

$$
\left|\begin{array}{lllll}
a_{p-q+1} & a_{p-q+2} & \cdot & \cdot & a_{p} \\
a_{p-q+2} & a_{p-q+3} & \cdot & \cdot & a_{p+1} \\
\cdot & \cdot & \cdot & \cdot & \cdot \\
\cdot & \cdot & \cdot & \cdot & \cdot \\
a_{p} & a_{p+1} & \cdot & \cdot & a_{p+q-1}
\end{array}\right| \neq 0,
$$

where $a_{i}=0$ for $i<0$. If this is satisfied we write $f \in \mathcal{D}_{p, q}(\zeta)$. For $f \in \mathcal{D}_{p, q}(\zeta)$ the Padé approximant

$$
[f ; p / q]_{\zeta}(z)=\frac{A(f, \zeta)(z)}{B(f, \zeta)(z)}
$$


is given by the following Jacobi formula

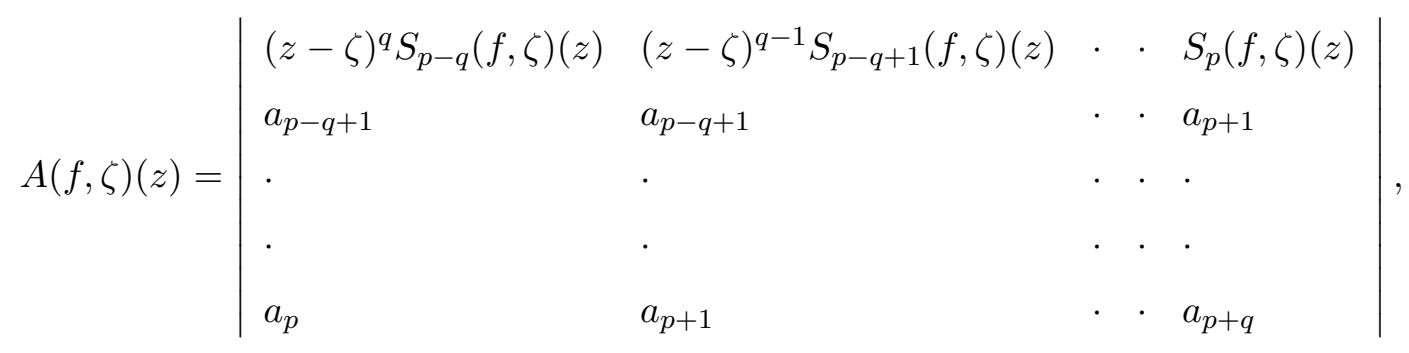

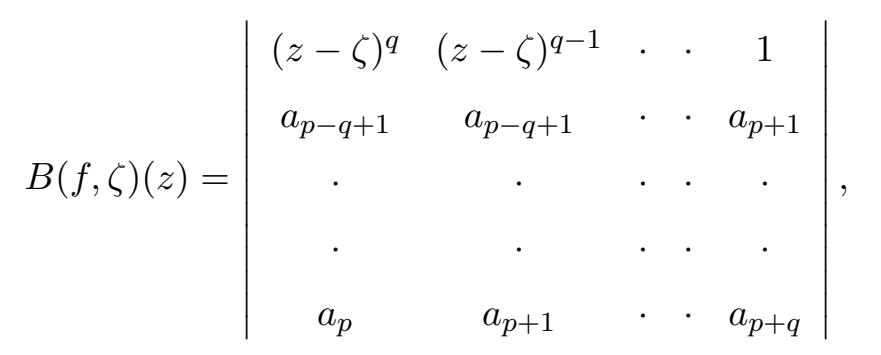

with (see [1])

$$
S_{k}(f, \zeta)(z)=\left\{\begin{array}{cc}
\sum_{\nu=0}^{k} a_{\nu}(z-\zeta)^{\nu}, & \text { if } \quad k \geq 0 \\
0, & \text { if } \quad k<0 .
\end{array}\right.
$$

If $A(f, \zeta)(z)$ and $B(f, \zeta)(z)$ are given by the previous Jacobi formula and do not have a common zero in a set $K$ we write $f \in E_{p, q, \zeta}(K)$. Equivalently

$$
|A(f, \zeta)(z)|^{2}+|B(f, \zeta)(z)|^{2} \neq 0
$$

for all $z \in K$. For $K$ compact this is equivalent to the existence of a $\delta>0$ such that

$$
|A(f, \zeta)(z)|^{2}+|B(f, \zeta)(z)|^{2}>\delta
$$

for all $z \in K$. We will also use the following ([1] Th. 1.4.4 page 30).

Proposition 2.2. Let $\phi(z)=\frac{A(z)}{B(z)}$ be a rational function, where the polynomials $A$ and $B$ do not have any common zero in $\mathbb{C}$. Let $\operatorname{deg} A(z)=k$ and $\operatorname{deg} B(z)=\lambda$. Then for every $\zeta \in \mathbb{C}$ such that $B(\zeta) \neq 0$ we have the following:

$$
\begin{gathered}
\quad \phi \in \mathcal{D}_{k, \lambda}(\zeta), \\
\phi \in \mathcal{D}_{p, \lambda}(\zeta) \text { for all } p>k, \\
\phi \in \mathcal{D}_{k, q}(\zeta) \text { for all } q>\lambda .
\end{gathered}
$$

In all these cases $\phi$ coincides with its corresponding Padé approximant, that is, $[\phi ; k / \lambda]_{\zeta}(z) \equiv \phi(z)$ and $[\phi ; p / \lambda]_{\zeta}(z) \equiv \phi(z)$ for all $p>k$ and $[\phi ; k / q]_{\zeta}(z) \equiv \phi(z)$ for $q>\lambda$. 
We shall also make use of Lemma 1 from [10] which states the following.

Proposition 2.3. Let $\Omega \subset \mathbb{C}$ be an open set. We suppose that the number of components of $\Omega$ is locally finite. Then there exists a sequence $K_{m} \subset \mathbb{C} \backslash \Omega m=1,2, \ldots$ of compact sets such that $K_{m} \cap \Omega=\emptyset$ with $K_{m}^{c}$ connected such that for every $K \subset \mathbb{C} \backslash \Omega$ compact set with $K^{c}$ connected there exists $m=1,2, \ldots$ with $K \subset K_{m}$.

Moreover, a slight modification at the proof of the above proposition that appears in [10] gives the following result.

Proposition 2.4. Let $\Omega \subseteq \mathbb{C}$ be an open set. We suppose that the number of components of $\Omega$ is locally finite. Then there exists a sequence $K_{m} \subset \mathbb{C} \backslash \bar{\Omega}, m=1,2, \ldots$ of compact sets with $K_{m}^{c}$ connected such that for every compact set $K \subset \mathbb{C} \backslash \bar{\Omega}$ with $K^{c}$ connected there exists $m=1,2, \ldots$ with $K \subset K_{m}$.

Definition 2.5. Let $\Omega \subseteq \mathbb{C}$. We say that $\left(L_{n}\right)_{n \in \mathbb{N}}$ is an exhausting sequence of compact sets in $\Omega$ if $\Omega=\bigcup_{n \in \mathbb{N}} L_{n}$ and for every $n \in \mathbb{N}$, it holds that $L_{n} \subset L_{n+1}^{0}$, where the interior is taken with respect to the relative topology of $\Omega$.

Proposition 2.6. If $\left(L_{n}\right)_{n \in \mathbb{N}}$ is an exhausting sequence of compact sets in $\Omega \subseteq \mathbb{C}$, then if $J \subset \Omega$ is a compact set, there exists $N \in \mathbb{N}$ such that $J \subset L_{N}$.

Proof. It is $J \subset \Omega=\bigcup_{n \in \mathbb{N}} L_{n} \subset \bigcup_{n \geq 2} L_{n}^{0}$ and therefore there exists $N \in \mathbb{N}$ such that $J \subset \bigcup_{n=2}^{N} L_{n}^{0}$, because $\zeta$ is compact. But $\left(L_{n}\right)_{n \in \mathbb{N}}$ is an increasing sequence of compact sets and therefore it holds $\zeta \subset \bigcup_{n=2}^{N} L_{n}^{0}=L_{N}^{0} \subset L_{N}$, as we wanted.

Proposition 2.7. If $\Omega \subseteq \mathbb{C}$ is locally compact then there exists an exhausting sequence of compact sets in $\Omega$.

Proof. Let $\widetilde{B}$ be a countable basis for $\Omega$ and consider $V=\{B \in \widetilde{B} \mid \bar{B}$ is compact $\}$.

Then $V$ is a basis since for any open $\mathcal{U}$ and $x \in \mathcal{U}$ we choose a compact neighborhood $N$ of $x$ inside $U$ and because $\widetilde{B}$ is a basis there exists $B \in \widetilde{B}$ such that $x \in B \subset N$; this implies $\bar{B} \subset N$ which yields that $B$ is compact and we found a $B \in V$ such that $x \in B \subset \mathcal{U}$. 
Therefore, we may assume that we have a countable basis $V=\left\{V_{n} \mid n \in \mathbb{N}\right\}$ where $\bar{V}_{n}$ is compact for every $n \in \mathbb{N}$. We define now the exhausting sequence of compact sets as follows.

We put $L_{1}=\bar{V}_{1}$. Since $V$ covers the compact set $L_{1}$ there exist $i_{1} \in \mathbb{N}$ such that $L_{1} \subset V_{1} \cup V_{2} \cup \cdots \cup V_{i_{1}}$.

Denoting $D_{1}$ the right hand side of the inclusion above, we put $L_{2}=\bar{V}_{1} \cup \bar{V}_{2} \cup$ $\cdots \cup \bar{V}_{i_{2}}=\bar{D}_{1}$.

This set is compact as a finite union of compacts.

Since $D_{1} \subset L_{2}$ and $D_{1}$ is open we get $D_{1} \subset L_{2}^{0}$, which gives $L_{1} \subset L_{2}^{0}$.

Next we choose $i_{2}>i_{1}$ such that $L_{2} \subset V_{1} \cup V_{2} \cup \cdots \cup V_{i_{2}}$, denote $D_{2}=V_{1} \cup V_{2} \cup \cdots \cup V_{i_{2}}$ and put $L_{3}=\bar{D}_{2}=\bar{V}_{1} \cup \bar{V}_{2} \cup \cdots \cup \bar{V}_{i_{2}}$. As before $L_{3}$ is compact and $L_{2} \subset L_{3}^{0}$.

Continuing this process we construct the sequence $\left(L_{n}\right)_{n \in \mathbb{N}}$ which clearly covers $\Omega$ and for every $n \in \mathbb{N}$ it holds $L_{n} \subset L_{n+1}^{0}$.

Proposition 2.8. Let $\Omega$ be an open set and $K \subseteq \mathbb{C}$ a compact subset of $\bar{\Omega}$. Then there exists a compact subset $K^{\prime}$ of $\bar{\Omega}$ such that

(i) $K \cap \partial \Omega=K^{\prime} \cap \partial \Omega$

(ii) $K \subseteq K^{\prime}$ and

(iii) Every connected component of $\{\infty\} \cup\left(\mathbb{C} \backslash K^{\prime}\right)$ contains a point from $\{\infty\} \cup(\mathbb{C} \backslash \bar{\Omega})$.

Proof. It is enough to show that if $\left(L_{n}\right)_{n \in I}, I \subseteq N$ are the bounded connected components of $\{\infty\} \cup(\mathbb{C} \backslash K)$ that are fully contained in $\Omega$, then the set $K^{\prime}=\left(\bigcup_{n \in I} L_{n}\right) \cup K$ is compact.

One can see that for every $n \in \mathbb{N}, \partial L_{n} \subset K$. This fact together with the fact that $K$ is bounded, yields that the union $\bigcup_{n \in I} L_{n}$ must also be bounded. Therefore, it is enough to show that $K^{\prime}$ is closed. Let $\left(x_{n}\right)_{n \in \mathbb{N}} \subset K^{\prime}$ with $x_{n} \rightarrow x$.

Since $K^{\prime} \subset \bar{\Omega}, x \in \bar{\Omega}$. Assume now that $x \notin K^{\prime}$. Then of course $x \notin K$ and since $K$ is compact, eventually $x_{n} \in \bigcup_{N \in I} L_{N}$. Moreover, since $\bar{L}_{n}=L_{n} \cup \partial L_{n} \subset L_{n} \cup K \subset K^{\prime}$, it is also true that $\left(x_{n}\right)_{n \in \mathbb{N}}$ does not have a whole subsequence inside one $L_{N}, N \in I$. Therefore $\operatorname{dist}\left(x, L_{m_{n}}\right) \rightarrow 0$, as $n \rightarrow+\infty$, for a subsequence $\left(L_{m_{n}}\right)_{n \in \mathbb{N}}$ of $\left(L_{n}\right)_{n \in \mathbb{N}}$. But this means $\operatorname{dist}\left(x, \partial L_{m_{n}}\right) \rightarrow 0$ as $n \rightarrow+\infty$. One can prove the above fact by observing that if $\lambda \in L_{N}$ for some $N \in \mathbb{N}$ then there exist $\lambda^{\prime} \in \partial L_{N}$ with $\left|\lambda^{\prime}-x\right| \leq$ 
$|\lambda-x|$, because the line segment $[\lambda, x]$ is a connected set and therefore the equality

$$
[\lambda, x]=\left(([\lambda, x]) \cap L_{N}^{0}\right) \cup\left([\lambda, x] \cap \partial L_{N}\right) \cup\left([\lambda, x] \cap\left(\mathbb{C} \backslash L_{N}\right)\right)
$$

yields $[\lambda, x] \cap \partial L_{N} \neq \emptyset$.

Now, the fact that $\operatorname{dist}\left(x, \partial L_{w_{n}}\right) \rightarrow 0$, as $n \rightarrow+\infty$ combined with $\partial L_{m_{n}} \subset K$ yields $\operatorname{dist}(x, K)=0$, which is a contradiction since $K$ is closed and $x \notin K$.

Corollary 2.9. Let $\Omega$ be an open set and $\left(L_{n}\right)_{n \in \mathbb{N}}$ be an increasing sequence of compact sets in $\bar{\Omega}$ such that

(i) For every $n \in \mathbb{N}, \overline{\left(L_{n} \cap \Omega\right)}=L_{n}$.

(ii) For every compact set $J$ in $\Omega$ there exists $N \in \mathbb{N}$ such that $J \subset L_{N}$.

Then, there exists an increasing sequence $\left(L_{n}^{\prime}\right)_{n \in \mathbb{N}}$ of compact sets in $\bar{\Omega}$ such that

1) $\left(L_{n}^{\prime}\right)_{n \in \mathbb{N}}$ satisfy conditions (i), (ii).

2) For every $n \in \mathbb{N}$ it holds $L_{n} \subseteq L_{n}^{\prime}, L_{n} \cap \partial \Omega=L_{n}^{\prime} \cap \partial \Omega$ and every connected component of $\{\infty\} \cup\left(\mathbb{C} \backslash L_{n}^{\prime}\right)$ contains a point from $\{\infty\} \cup(\mathbb{C} \backslash \bar{\Omega})$.

Proof. From Proposition 2.8 for every $L_{n}, n \in \mathbb{N}$, we can find a compact set $L_{n}^{\prime}$ such that $L_{n} \subseteq L_{n}^{\prime}, L_{n} \cap \partial \Omega=L_{n}^{\prime} \cap \partial \Omega$ and every connected component of $\{\infty\} \cup\left(\mathbb{C} \backslash L_{n}^{\prime}\right)$ contains a point from $\{\infty\} \cup(\mathbb{C} \backslash \bar{\Omega})$.

One can check that the sequence $\left(L_{n}^{\prime}\right)_{n \in \mathbb{N}}$ satisfy the conditions we want.

\section{Main Results}

Let $\Omega$ be an open set, $\Omega \subseteq \mathbb{C}$ and $L_{n}, n \in \mathbb{N}$, be an increasing sequence of compact subsets of $\bar{\Omega}$. For this sequence we also assume firstly that for every $n \in \mathbb{N}$ it holds $\overline{\left(L_{n}, \cap \Omega\right)}=L_{n}$, secondly that each connected component of $\{\infty\} \cup\left(\mathbb{C} \backslash L_{n}\right)$ contains a connected component of $\{\infty\} \cup(\mathbb{C} \backslash \bar{\Omega})$ and finally that every compact set $J$ inside of $\Omega$ is contained in some $L_{m}$, for a natural number $m$.

Let $T^{\infty}(\Omega)=T^{\infty}\left(\Omega,\left(L_{n}\right)_{n \in \mathbb{N}}\right)$ be the space of all analytic functions $f \in H(\Omega)$, such that, for every derivative $f^{(\ell)}, \ell \in \mathbb{N}_{0}$, and every $L_{n}, n \in \mathbb{N}$ the restriction $\left.f^{(\ell)}\right|_{\left(L_{n} \cap \Omega\right)}$ is uniformly continuous and therefore it extends continuously on $\overline{\left(L_{n} \cap \Omega\right)}=L_{n}$.

We endow this space with the seminorms $\sup _{z \in L_{n}}\left|f^{(\ell)}(z)\right|, \ell \in \mathbb{N}_{0}, n \in \mathbb{N}$. Then, it becomes a Frechet space, containing the rational functions with poles off the union 
$\bigcup_{n \in \mathbb{N}} L_{n}$. Consider now, $Y^{\infty}(\Omega)$ the closure of the set of all the rational functions with poles off $\bigcup_{n \in \mathbb{N}} L_{n}$. As a closed subset of a complete space, $Y^{\infty}(\Omega)$ is also a complete space and therefore Baire's theorems is at our disposal.

Based on the above facts we are now ready for our first theorem.

Theorem 3.1. Let $F \subset \mathbb{N} \times \mathbb{N}$ be a set that contains a sequence $\left(\widetilde{p}_{n}, \widetilde{q}_{n}\right), n=1,2, \ldots$ such that $\widetilde{p}_{n} \rightarrow+\infty$ and $\widetilde{q}_{n} \rightarrow+\infty$ and let $\Omega \subseteq \mathbb{C}$ be an open set. Let also $K \subseteq \mathbb{C}$ be a compact set such that $K \cap L_{n}=\emptyset$ for every $n \in \mathbb{N}$ and let $m \in \mathbb{N}$ be a fixed natural number.

Then there exists $f \in Y^{\infty}(\Omega)$ such that: for every rational function $h$ there exists a sequence $\left(p_{n}, q_{n}\right) \in F(n=1,2, \ldots)$ with the following properties:

(i) $f \in D_{p_{n}, q_{n}}(\zeta) \cap E_{p_{n}, q_{n}, \zeta}\left(L_{m} \cup K\right)$, for every $\zeta \in L_{m}, n \in \mathbb{N}$.

(ii) For every $\ell \in \mathbb{N}$, $\sup _{\zeta \in L_{m}} \sup _{z \in L_{m}}\left|\left[f ; p_{n} / q_{n}\right]_{\zeta}^{(\ell)}(z)-f^{(\ell)}(z)\right| \rightarrow 0$, as $n \rightarrow+\infty$.

(iii) $\sup _{\zeta \in L_{m}} \sup _{z \in K} \chi\left(\left[f ; p_{n} / q_{n}\right]_{\zeta}(z), h(z)\right) \rightarrow 0$, as $n \rightarrow+\infty$.

The set of such functions $f \in Y^{\infty}(\Omega)$ is dense and $G_{\delta}$ in $Y^{\infty}(\Omega)$.

Proof. Let $\left(f_{j}\right)_{j \in \mathbb{N}}$ be an enumeration of the rational functions having coefficients of the numerator and denominator from $\mathbb{Q}+i \mathbb{Q}$.

We denote $\mathcal{U}^{K}(m)$ the set of all functions in $Y^{\infty}(\Omega)$ that satisfy the properties (i), (ii) and (iii).

We will prove that $\mathcal{U}^{K}(m)$ is a $G_{\delta}$-dense subset of $Y^{\infty}(\Omega)$ and therefore $\mathcal{U}^{K}(m) \neq \emptyset$.

For $j, s \in \mathbb{N}$ and $(p, q) \in F$ we define:

$$
\begin{aligned}
E(j, p, q, s)= & \left\{f \in Y^{\infty}(\Omega) \mid f \in D_{p, q}(\zeta) \cap E_{p, q, \zeta}(K) \text { for all } \zeta \in L_{m}\right. \\
& \text { and } \left.\sup _{\zeta \in L_{m}} \sup _{z \in K} \chi\left([f ; p / q]_{\zeta}(z), f_{j}(z)\right)<1 / s\right\}
\end{aligned}
$$

and,

$$
\begin{aligned}
& T(p, q, s)=\left\{f \in Y^{\infty}(\Omega) \mid f \in D_{p, q}(\zeta) \cap E_{p, q, \zeta}\left(L_{m}\right) \text { for all } \zeta \in L_{m}\right. \\
&\text { and } \left.\sup _{\zeta \in L_{m}} \sup _{z \in L_{m}}\left|[f ; p / q]_{\zeta}^{(\ell)}(z)-f^{(\ell)}(z)\right|<1 / s \text { for } \ell=0,1, \ldots, s\right\} .
\end{aligned}
$$

Proposition 2.1 and the definition of $Y^{\infty}(\Omega)$ easily implies that

$$
U^{K}(m)=\bigcap_{j, s=1}^{+\infty} \bigcup_{(p, q) \in F}(E(j, p, q, s) \cap T(p, q, s)) .
$$


To prove that $U^{K}(m)$ is a $G_{\delta}$-dense in the $Y^{\infty}(\Omega)$-topology, it is enough to prove that for every $j, s=1,2, \ldots$ and $(p, q) \in F$ the sets $E(j, p, q, s), T(p, q, s)$ are open in $Y^{\infty}(\Omega)$ and that for every $j$ and $s$ from $\mathbb{N}^{*}$, the set $\bigcup_{(p, q) \in F}(E(j, p, q, s) \cap T(p, q, s))$ is dense in $Y^{\infty}(\Omega)$.

For, let $j, s \in \mathbb{N}$ and $(p, q) \in F$.

We first prove that the set $E(j, p, q, s)$ is open in $Y^{\infty}(\Omega)$. Indeed, let $f \in E(j, p, q, s)$ and let $g \in Y^{\infty}(\Omega)$ be such that $\sup _{z \in L_{m}}\left|f^{(t)}(z)-g^{(t)}(z)\right|<a$ for $t=0,1,2, \ldots, p+q+1$ (relation (1)).

The number $a>0$ will be determined later on. It is enough to prove that if $a$ is small enough then $g \in E(j, p, q, s)$.

The Hankel determinants defining $D_{p, q}(\zeta)$ for $f$ depend continuously on $\zeta \in L_{m}$; thus, there exists $\delta>0$ such that the absolute values of the corresponding Hankel determinants are greater than $\delta>0$, for every $\zeta \in L_{m}$, because $f \in D_{p, q}(\zeta)$ for $\zeta \in L_{m}$ and because $L_{m}$ is compact.

From relation (1) we can control the first $p+q+1$ Taylor coefficients of $g$ and by making $a>0$ small enough one can get the Hankel determinants that define $D_{p, q}(\zeta)$ to have absolute value at least $\delta / 2>0$.

Therefore, $g$ will belong to $D_{p, q}(\zeta)$ for every $\zeta \in L_{m}$. Now we consider the Padé approximants of $f, g$ according to the Jacobi formula (see preliminaries)

$$
[f ; p / q]_{\zeta}(z)=\frac{A(f, \zeta)(z)}{B(f, \zeta)(z)} \text { and }[g ; p / q]_{\zeta}(z)=\frac{A(g, \zeta)(z)}{B(g, \zeta)(z)}
$$

Now $|A(f, \zeta)(z)|^{2}+|B(f, \zeta)(z)|^{2}$ vary continuously with respect to $(z, \zeta) \in K \times L_{m}$, because of the Jacobi formula. So, there is a $\delta^{\prime}>0$ such that:

$$
|A(f, \zeta)(z)|^{2}+|B(f, \zeta)(z)|^{2} \geq \delta^{\prime}, \quad \text { for all } \zeta \in L_{m} \text { and } z \in K
$$

Now again from the Jacobi formula, if $a$ is small enough, one gets:

$$
|A(g, \zeta)(z)|^{2}+|B(g, \zeta)(z)|^{2} \geq \delta^{\prime} / 2, \text { for all } \zeta \in L_{m} \text { and } z \in K \text {. }
$$

This yields that $g \in E_{p, q, \zeta}(K)$ for every $\zeta \in L_{m}$. For the rest it is enough to show that if $a$ is small enough then $\sup _{\zeta \in L_{m}} \sup _{z \in K} \chi\left([g ; p / q]_{\zeta}(z),[f ; p / q]_{\zeta}(z)\right)$ can become less than $\frac{1}{s}-\sup _{\zeta \in L} \sup _{z \in K} \chi\left([f ; p / q]_{\zeta}(z), f_{j}(z)\right) \equiv \gamma>0$. By taking $a$ small as before we have 
that $|A(f, \zeta)(z)|^{2}+|B(f, \zeta)(z)|^{2}>\delta^{\prime}$ and for every $\zeta \in L_{m}$ and $z \in K$ we have $|A(g, \zeta)(z)|^{2}+|B(g, \zeta)(z)|^{2}>\delta^{\prime} / 2$.

It follows that

$$
\begin{aligned}
& \chi\left([f ; p / q]_{\zeta}(x),[g ; p / q]_{\zeta}(z)\right) \\
& =\frac{|A(f, \zeta)(z) B(g, \zeta)(z)-A(g, \zeta)(z) B(f, \zeta)(z)|}{\sqrt{|A(f, \zeta)(z)|^{2}+|B(f, \zeta)(z)|^{2}} \sqrt{|A(g, \zeta)(z)|^{2}+|B(g, \zeta)(z)|^{2}}} \\
& \quad \leq \frac{\sqrt{2}}{\delta^{\prime}}|A(f, \zeta)(z) B(g, \zeta)(z)-A(g, \zeta)(z) B(f, \zeta)(z)|
\end{aligned}
$$

for all $\zeta \in L_{m}$ and $z \in K$, which easily yields the result, because the last expression can become as small as we want to, uniformly for all $\zeta \in L_{m}, z \in K$. Thus, we proved that $E(j, p, q, s)$ is open.

Next, we prove that $T(p, q, s)$ is also open in $Y^{\infty}(\Omega)$. Let $f$ be a function in $T(p, q, s)$ and let $g$ be a function in $Y^{\infty}(\Omega)$ such that: $\sup _{z \in L_{m}^{\prime}}\left|f^{(t)}(z)-g^{(t)}(z)\right|<a$, for $t=0,1,2, \ldots, \max (s, p+q+1)$, where $a>0$ will be determined later on.

In the same way as before one deduces that by making " $a$ " small enough it follows that $g \in D_{p, q}(\zeta) \cap E_{p, q, \zeta}\left(L_{m}\right)$, for all $\zeta \in L_{m}$.

Now $f(z) \in \mathbb{C}$, for each $z \in L_{m}$. It follows that for all $\zeta \in L_{m}, z \in L_{m}$ we have $[f ; p / q]_{\zeta}(z) \in \mathbb{C}$. Therefore, $B(f, \zeta)(z) \neq 0$, where $B$ is given by the Jacobi formula.

So there is a $\delta^{\prime \prime}>0$ such that $\delta^{\prime \prime}<1$ and $|B(f, \zeta)(z)|>\delta^{\prime \prime}$ for all $\zeta \in L_{m}, z \in L_{m}$, because $L_{m} \times L_{m}$ is compact.

By making " $a$ " small enough, by continuity one can get

$$
|B(g, \zeta)(z)|>\frac{\delta^{\prime \prime}}{2} \text { for all } \zeta \in L_{m}, \quad z \in L_{m}
$$

For $\ell \in\{0,1, \ldots, s\}$ it holds

$$
\begin{aligned}
\sup _{\zeta \in L_{m}} \sup _{z \in L_{m}}\left|[g ; p / q]_{\zeta}^{(\ell)}(z)-g^{(\ell)}(z)\right| \leq & \sup _{z \in L_{m}}\left|f^{(\ell)}(z)-g^{(\ell)}(z)\right| \\
& +\sup _{\zeta \in L_{m}} \sup _{z \in L_{m}}\left|f^{(\ell)}(z)-[f ; p / q]_{\zeta}^{(\ell)}(z)\right| \\
& +\sup _{\zeta \in L_{m}} \sup _{z \in L_{m}}\left|[g ; p / q]_{\zeta}^{(\ell)}(z)-[f ; p / q]_{\zeta}^{(\ell)}(z)\right| .
\end{aligned}
$$

The first term obviously get small as " $a$ " gets small. Since the second term is fixed and less than $1 / s$ we must control only the last term.

But the Jacobi denominators of $[f ; p / q]_{\zeta}^{(\ell)}(z)$ and $[g ; p / q]_{\zeta}^{(\ell)}(z)$ are bounded below from $\left(\delta^{\prime \prime}\right)^{\ell+1}$ and $\left(\delta^{\prime \prime} / 2\right)^{\ell+1}$ respectively for $\ell=0,1, \ldots, s$. 
Thus, the last term can get as small as we want for all $\ell=0,1, \ldots, s$, if $a$ is small enough. We are done.

Finally, we prove that for all $j, s \in \mathbb{N}$ the set $\bigcup_{(p, q) \in F}(E(j, p, q, s) \cap T(p, q, s))$ is dense in $Y^{\infty}(\Omega)$.

Let $g$ be a function inside $Y^{\infty}(\Omega), \varepsilon>0$ and $r, N \in \mathbb{N}$. Because of the definition of $Y^{\infty}(\Omega)$ we can assume without loss of generality that $g$ is a rational function with poles off $\bar{\Omega}$, as we want to approximate $g$ on $L_{r}$ and every connected component of $\{\infty\} \cup\left(\mathbb{C} \backslash L_{r}\right)$ contains a point from $\{\infty\} \cup(\mathbb{C} \backslash \bar{\Omega})$.

To prove what we want to, we have to find a function $f \in Y^{\infty}(\Omega)$ and a pair $(p, q) \in F$ such that

(1) $f \in D_{p, q}(\zeta) \cap E_{p, q, \zeta}\left(K \cup L_{m}\right)$, for all $\zeta \in L_{m}$.

(2) $\sup _{\zeta \in L_{m}} \sup _{z \in K} \chi\left([f ; p / q]_{\zeta}(z), f_{j}(z)\right)<\frac{1}{s}$.

(3) $\sup _{\zeta \in L_{m}} \sup _{z \in L_{m}}\left|f^{(\ell)}(z)-[f ; p / q]_{\zeta}^{(\ell)}(z)\right|<\frac{1}{s}$, for $\ell=0,1, \ldots, s$.

(4) $\sup _{z \in L_{r}}\left|f^{(t)}(z)-g^{(t)}(z)\right|<\varepsilon$, for $t=0,1, \ldots, N$.

Without loss of generality we may assume that $r>m$ or equivalently $L_{r} \supset L_{m}$.

Let $w: L_{r} \cup K \rightarrow \mathbb{C}$ such that $q(z)=\left\{\begin{array}{ll}f_{j}(z) & z \in K \\ g(z), & z \in L_{r}\end{array}\right.$.

Now, let $\mu$ be the sum of the principal parts of the poles of the rational function $f_{j}$ that belong to $K$. Then $(\omega-\mu)$ is holomorphic in a neighborhood of $\left(L_{r}^{\prime} \cup K\right)$. Combining Runge's with Weierstrass Theorems we conclude that there exists a rational function $\frac{\widetilde{A}(z)}{\widetilde{B}(z)}$ with poles out of $\left(L_{r}^{\prime} \cup K\right)$, approximating $(\omega-\mu)$ uniformly on $L_{r}^{\prime} \cup$ $K$ with respect to the euclidean metric and in the level of all derivatives of order from zero to $N$. That implies that the function $\frac{A(z)}{B(z)}=\mu(z)+\frac{\widetilde{A}(z)}{\widetilde{B}(z)}$ approximates $f_{j}(z)$, uniformly on $K$ with respect to the chordal distance, and also that $\left(\frac{A(z)}{B(z)}\right)^{(\ell)}$ approximates the function $(g(z))^{(\ell)}$ uniformly on $L_{r}^{\prime}$, with respect to the euclidean metric. Obviously, we can assume that the greatest common divisor of $A(z)$ and $B(z)$ is equal to one.

From our assumption on $F$, there exists a pair $(p, q) \in F$ such that $p>\operatorname{deg} A, \operatorname{deg} B$ and $q>\operatorname{deg} B$. We consider the function $\frac{A(z)}{B(z)}+d z^{T}=\frac{A(z)+d \cdot z^{T} \cdot B(z)}{B(z)}$ where 
$T=p-\operatorname{deg} B$ and $d$ is different from zero. Now, it is easy to see that $\operatorname{gcd}(A(z)+$ $\left.d z^{T} B(z), B(z)\right)$ equals again to one. Thus, according to Proposition 2.2 it holds that for all $\zeta \in \mathbb{C}$ such that $B(\zeta) \neq 0$ the rational function $\frac{A(z)+d z^{T} B(z)}{B(z)}$ belongs to $D_{p, q}(\zeta)$ and also $\left[\frac{A(z)+d z^{T} B(z)}{B(z)} ; p / q\right]_{\zeta}(z)=\frac{A(z)+d z^{T} B(z)}{B(z)}$. In particular the above holds for all $\zeta \in L_{m}^{\prime}$, because $B(\zeta) \neq 0$ for all $\zeta \in L_{r}^{\prime}$.

We distinguish the case $B(z) \neq 0$ for all $z \in \bigcup_{n} L_{n}$ and the case where $B$ has zeros in $\left(\bigcup_{n \in \mathbb{N}} L_{n}\right) \backslash L_{r}$. First assume that $B(z) \neq 0$ for all $z \in \bigcup_{n} L_{n}$. In this case we set $f(z)=\frac{A(z)}{B(z)}+d z^{T}$, and by selecting $d$ with $|d|$ small enough, we are done.

In the second case, since every component of $\widetilde{\mathbb{C}} \backslash L_{r}$ contains a point from $\widetilde{\mathbb{C}} \backslash \bar{\Omega}$, there exists a rational function that belongs to $Y^{\infty}(\Omega)$, call it $f$, such that every finite set of derivatives $f^{(\ell)}$ are close to $\left(\frac{A(z)+d z^{T}}{B(z)}\right)^{(\ell)}$ uniformly on $L_{r}$. This follows immediately from Runge's and Weierstrass Theorems and also from the fact that $B$ has finitely many roots outside $L_{r}$ and thus in a positive distance from $L_{r}$.

It is easy to see that $f$ fulfills all requirements in the same way as $\frac{A(z)+d z^{T} B(z)}{B(z)}$ does except from the fact that maybe $[f ; p / q]_{\zeta}(z) \neq f(z)$.

But the following is true:

$$
\begin{aligned}
\sup _{\zeta \in L} \sup _{z \in \Delta}\left|[f ; p / q]_{\zeta}^{(\ell)}(z)-f^{(\ell)}(z)\right| \leq & \sup _{z \in \Delta}\left|f^{(\ell)}(z)-h^{(\ell)}(z)\right| \\
& +\sup _{\zeta \in L} \sup _{z \in \Delta}\left|h^{(\ell)}(z)-[h ; p / q]_{\zeta}^{(\ell)}(z)\right| \\
& +\sup _{\zeta \in L} \sup _{z \in \Delta}\left|[h ; p / q]_{\zeta}^{(\ell)}(z)-[f ; p / q]_{\zeta}^{(\ell)}(z)\right| .
\end{aligned}
$$

with $h(z)=\frac{A(z)+d z^{T} B(z)}{B(z)}$.

Now, as $p, q$ are fixed and we can control any finite set of derivatives of $f$, we can also control any finite set of Taylor coefficients of $f$. Thus, we can make the first and the last term of the right-hand side expression in $(*)$ as small as we want and we are done.

This completes the proof of the Theorem.

Now, if we define as above $\mathcal{U}^{K}(m)$ the set of all the functions that satisfy the requirements of Theorem 3.1 we have shown that for every $m \in \mathbb{N}$ and $K \subseteq \mathbb{C}$ compact such that $K \cap L_{n}=\emptyset$, for every $n \in \mathbb{N}, \mathcal{U}^{K}(m)$ is a $G_{\delta^{-}}$dense subset of $Y^{\infty}(\Omega)$. There- 
fore, if we have a sequence $\left(K_{r}\right)_{r \in \mathbb{N}}$ of such compact sets and apply Baire's Theorem at $\bigcap_{r, m \in \mathbb{N}} \mathcal{U}^{K_{r}}(m)$ we get the following result.

Theorem 3.2. Let $F \subset \mathbb{N} \times \mathbb{N}$ be a set that contains a sequence $\left(\widetilde{p}_{n}, \widetilde{q}_{n}\right) \in F$ such that $\widetilde{p}_{n} \rightarrow+\infty, \widetilde{q}_{n} \rightarrow+\infty$ and let $\Omega \subseteq \mathbb{C}$ be an open set. Then there exists a function $f \in Y^{\infty}(\Omega)$ such that for every rational function $h$ and $K_{s}$ member of the sequence $\left(K_{r}\right)_{r \in \mathbb{N}}$ there exists a sequence $\left(p_{n}, q_{n}\right) \in F, n=1,2, \ldots$ with the following properties:

For every $m \in \mathbb{N}$ there exists $n(m) \in \mathbb{N}$ such that

(i) $f \in D_{p_{n}, q_{n}}(\zeta) \cap E_{p_{n}, q_{n} \zeta}\left(K_{s} \cup L_{m}\right)$, for every $n \geq n(m)$

(ii) For every $\ell \in \mathbb{N}$, $\sup _{\zeta \in L_{m}} \sup _{z \in L_{M}}\left|\left[f ; p_{n} / q_{n}\right]_{\zeta}^{(\ell)}(z)-f^{(\ell)}(z)\right| \rightarrow 0$, as $n \rightarrow+\infty$ and

(iii) $\sup _{\zeta \in L_{m}} \sup _{z \in K_{s}} \chi\left(\left[f ; p_{n} / q_{n}\right]_{\zeta}(z), h(z)\right) \rightarrow 0$, as $n \rightarrow+\infty$.

The set of such functions $f \in Y^{\infty}(\Omega)$ is dense and $G_{\delta}$ in $Y^{\infty}(\Omega)$.

Further, if we start with an open set $\Omega$ such that $\{\infty\} \cup(\mathbb{C} \backslash \bar{\Omega})$ is connected then we can get the following result.

Theorem 3.3. Let $\Omega$ be an open set such that $\{\infty\} \cup(\mathbb{C} \backslash \bar{\Omega})$ is connected and let $F$ be a subset of $\mathbb{N} \times \mathbb{N}$ that contains a sequence $\left(\widetilde{p}_{n}, \widetilde{q}_{n}\right), n=1,2, \ldots$ with $\widetilde{p}_{n} \rightarrow+\infty$.

Let also $K \subseteq \mathbb{C}$ be a compact set such that $K \cap L_{n}=\emptyset$ for every $n \in \mathbb{N}$ and let also $m \in \mathbb{N}$ be a fixed natural number.

Then there exists a function $f \in Y^{\infty}(\Omega)$ such that for every polynomial $p$ there exists a sequence $\left(p_{n}, q_{n}\right) \in F(n=1,2, \ldots)$ with the following properties:

(i) $f \in D_{p_{n}, q_{n}}(\zeta) \cap E_{p_{n}, q_{n}, \zeta}\left(L_{m} \cup K\right), \forall \zeta \in L_{m}$.

(ii) For every $\ell \in \mathbb{N}$, $\sup _{\zeta \in L_{m}} \sup _{z \in L_{m}}\left|\left[f ; p_{n} / q_{n}\right]^{(\ell)}(z)-f^{(\ell)}(z)\right| \rightarrow 0$, as $n \rightarrow+\infty$.

(iii) For every $\ell \in \mathbb{N}$, $\sup _{\zeta \in L_{m}} \sup _{z \in K}\left|\left[f ; p_{m} / q_{n}\right]_{\zeta}^{(\ell)}(z)-p^{(\ell)}(z)\right| \rightarrow 0$, as $n \rightarrow+\infty$.

The set of such functions $f \in Y^{\infty}(\Omega)$ is dense and $G_{\delta}$ in $Y^{\infty}(\Omega)$.

Proof. First we enumerate the polynomials with coefficients from $\mathbb{Q}+i \mathbb{Q},\left(\rho_{j}\right), j=$ $1,2, \ldots$ and we call $V^{K}(m)$ the set of the functions that satisfy the prerequisites of the Theorem 3.3 .

Now, we set for $j, s \in \mathbb{N}$ and $(p, q) \in \mathbb{F}$,

$$
\begin{gathered}
E(j, p, q, s)=\left\{f \in Y^{\infty}(\Omega) \mid f \in D_{p, q}(\zeta) \cap E_{p, q, \zeta}(K), \text { for every } \zeta \in L_{m}\right. \\
\left.\quad \text { and } \sup _{\zeta \in L_{m}} \sup _{z \in K}\left|\left[f_{i} p / q\right]_{\zeta}(z)-p_{j}(z)\right|<\frac{1}{s}\right\}
\end{gathered}
$$


and

$$
\begin{aligned}
& T(p, q, s)=\left\{f \in Y^{\infty}(\Omega) \mid f \in D_{p, q}(\zeta) \cap E_{p, q, \zeta}\left(L_{m}\right), \text { for every } \zeta \in L_{m}\right. \\
&\text { and } \left.\sup _{\zeta \in L_{m}} \sup _{z \in L_{m}}\left|[f ; p / q]_{\zeta}^{(\ell)}(z)-f^{\ell)}(z)\right|<\frac{1}{s} \text { for } \ell=0,1, \ldots, s\right\} .
\end{aligned}
$$

Then the definition of $V^{K}(m)$ yields that

$$
V^{K}(m)=\bigcap_{j, s=1}^{+\infty} \bigcup_{(p, q) \in F}(E(j, p, q, s) \cap T(p, q, s)) .
$$

Then, we prove that $V^{K}(m)$ is a $G_{\delta}$-dense subset of $Y^{\infty}(\Omega)$ and therefore $V^{K}(m) \neq \emptyset$, which is what we want to.

For, we prove that for every $i, s=1,2, \ldots$ and $(p, q) \in F$ the sets $E(j, p, q, s)$, $T(p, q, s)$ are open in $Y^{\infty}(\Omega)$ and that for every $j, s=1,2$ the set $\bigcup_{(p, q) \in F}(E(j, p, q, s) \cap$ $T(p, q, s))$ is dense in $Y^{\infty}(\Omega)$.

The proof that $E(j, p, q, s)$ and $T(p, q, s)$ are open in $Y^{\infty}(\Omega)$ is the same as in the corresponding of Theorem 3.1 and therefore is omitted.

To prove the density result let $i, s=1,2, \ldots$.

We will show that $\bigcup_{(p, q) \in F}\left(E(j, p, q, s) \cap T(p, q, s)\right.$ is dense in $Y^{\infty}(\Omega)$.

For, let $g$ be a rational function with poles off $\bar{\Omega}, \varepsilon>0, N \in \mathbb{N}$ and $L_{r}$ be a member of the sequence of compacts $\left(L_{n}\right)_{n \in \mathbb{N}}$ that defined $Y^{\infty}(\Omega)$. Because $\left(L_{n}\right)_{n \in \mathbb{N}}$ is an increasing sequence of compact sets we may assume that $L_{r} \supset L_{m}$ and because of the fact that $\{\infty\} \cup(\mathbb{C} \backslash \bar{\Omega})$ is connected and Runge's Theorem we may also assume that $q$ is a polynomial.

Now to prove what we want to, we need to find a function $f \in Y^{\infty}(\Omega)$ and a pair $(p, q) \in F$ such that

(i) $f \in D_{p, q}(\zeta) \cap E_{p, q, \zeta}\left(K \cup L_{m}\right), \quad \forall \zeta \in L_{m}$.

(ii) $\sup _{\zeta \in L_{m}} \sup _{z \in K}\left|[f ; p / q]_{\zeta}(z)-f_{j}(z)\right|<\frac{1}{s}$.

(iii) $\sup _{\zeta \in L_{m}} \sup _{z \in L_{m}}\left|[f ; p / q]_{\zeta}^{(\ell)}(z)-f^{(\ell)}(z)\right|<\frac{1}{s}$, for $\ell=0,1, \ldots, s$ and

(iv) $\sup _{z \in L_{r}}\left|f^{(t)}(z)-g^{(t)}(z)\right|<\varepsilon$, for $t=0,1, \ldots, N$.

Let $w: L_{r} \cup K \rightarrow \mathbb{C}$ defined by $w(z)=\left\{\begin{array}{cc}f ;(z), & z \in K \\ g(z), & z \in L_{r}\end{array}\right.$. Because of the fact that every connected component of $\{\infty\} \cup\left(\mathbb{C} \backslash L_{r}\right)$ contains a connected component 
of $\{\infty\} \cup(\mathbb{C} \backslash \bar{\Omega})$ and the last one is a connected set we have that $\{\infty\} \cup\left(\mathbb{C} \backslash L_{r}\right)$ is also connected.

Moreover, since $K^{c}$ is connected and $K \cap L_{n}=\emptyset$ is a compact set Runges and Weierstrass theorem yields the existence of a polynomial $h$ that approximates $w$ in the level of any finite set of derivatives on $K \cup L_{r}$. Now, there exist a pair $(p, q) \in F$ with $p>\operatorname{deg}(g)$.

Therefore, if we set $f(z)=g(z)+d z^{p}$, for $d \in \mathbb{C}$ with $|d| \neq 0$ small enough one can check with the help of Proposition 2.2 that $f(z)$ satisfies all the four conditions we want. This completes the proof of Theorem 3 .

Now, if we set $V^{K}(m)$, as in the above proof, for $K \subseteq \mathbb{C}$ a compact set with connected complement such that $K \cap L_{n}=\emptyset$, for every $n \in \mathbb{N}$ and $m \in \mathbb{N}$, the set of all functions $f \in Y^{\infty}(\Omega)$ that satisfy the requirements of Theorem 3 , we have shown that $V^{K}(m)$ is a $G_{\delta}$ and dense subset of $Y^{\infty}(\Omega)$. Therefore, by applying Baire's Theorem at $\bigcap_{\ell, m \in \mathbb{N}} V^{K_{\ell}}(m)$, where $\left(K_{\ell}\right)_{\ell \in \mathbb{N}}$ is a set of compact sets in $\mathbb{C}$ with connected complement such that $K_{\ell} \cap L_{n}=\emptyset$ for every $\ell, n \in \mathbb{N}$ we get the following result.

Theorem 3.4. Let $F \subset \mathbb{N} \times \mathbb{N}$ be a set that contains a sequence $\left(\widetilde{p}_{n}, \widetilde{q}_{n}\right) \in F$ such that $\widetilde{p}_{n} \rightarrow+\infty$ and let $\Omega \subseteq \mathbb{C}$ be an open set. Then there exists a function $f \in Y^{\infty}(\Omega)$ such that for every polynomial function $p$ and $K_{s}$ member of the sequence $\left(K_{\ell}\right)_{\ell \in \mathbb{N}}$ that we defined above, there exist a sequence $\left(p_{n}, q_{n}\right) \in F, n=1,2, \ldots$ with the following properties: For every $m \in \mathbb{N}$, there exists $n(m) \in \mathbb{N}$ such that:

(i) $f \in D_{p_{n}, q_{n}}(\zeta) \cap E_{p_{n}, q_{n}, \zeta}\left(K_{s} \cup L_{m}\right)$, for every $n \geq n(m)$.

(ii) For every $\ell \in \mathbb{N}$, $\sup _{\zeta \in L_{m}} \sup _{z \in L_{m}}\left|\left[f ; p_{n} / q_{n}\right]_{\zeta}^{(\ell)}(z)-f^{(\ell)}(z)\right| \rightarrow 0$.

(iii) $\sup \sup \left|\left[f ; p_{n} / q_{n}\right]_{\zeta}(z)-p(z)\right| \rightarrow 0$. $\zeta \in L_{m} z \in K_{s}$

The set of such functions $f \in Y^{\infty}(\Omega)$ is dense and $G_{\delta}$ in $Y^{\infty}(\Omega)$.

Remark 3.5. One can check that in the case where $\Omega$ is a simply connected domain and $L_{n} \cap \partial \Omega=\emptyset$, for every $n \in \mathbb{N}$, the proofs of Theorem 3.3, 3.4 can be easily modified to show that the results under this new hypothesis remain true.

\section{Applications}

We consider now different cases for the sequences $\left(L_{n}\right)_{n \in \mathbb{N}}$ and $\left(K_{r}\right)_{r \in \mathbb{N}}$ and we discuss what old or new results one can get from the theorems of Section 3 under these 
hypothesis.

4.1. Consider first the case where $\left(L_{n}\right)_{n \in \mathbb{N}}$ is an exhausting sequence of compact sets in $\Omega$, where $\Omega \subseteq \mathbb{C}$ is an open set and $K=\emptyset$.

Such a sequence $\left(L_{n}\right)_{n \in \mathbb{N}}$ exists because of the fact that $\Omega$ is an open set and of Proposition 2.7. Moreover, such a sequence can be easily modified as in Corollary 2.9 to give also an exhausting sequence of compact sets in $\Omega$ and to fulfill also the conditions to define the space $T^{\infty}(\Omega)=T^{\infty}\left(\Omega,\left(L_{n}\right)_{n \in \mathbb{N}}\right)$, that we introduced at Section 3. Furthermore, in this case $T^{\infty}(\Omega)$ becomes equal to $H(\Omega)$. But by Runge's Theorem the set of rational function with poles off $\Omega=\bigcup_{n} L_{n}$ is dense in $H(\Omega)$ which can be translated to $Y^{\infty}(\Omega)=H(\Omega)$, where $Y^{\infty}(\Omega)$ is also defined in Section 3. Now, under these hypothesis, Theorem 3.1 yields a result from [6], according to which generically every holomorphic function $f$ defined on an open set $\Omega$ can be approximated by a sequence of it's Padé approximants obtained from a set $\{[f ; p / q] \mid(p, q) \in F\}$ where $F$ is a subset of $\left(\mathbb{N} \times \mathbb{N}\right.$ that contains a sequence $\left(\widetilde{p}_{n}, \widetilde{q}_{n}\right), n=1,2, \ldots$ where $\widetilde{p}_{n} \rightarrow+\infty$, $\widetilde{q}_{n} \rightarrow+\infty$.

4.2. We consider now the case where $\left(L_{n}\right)_{n \in \mathbb{N}}$ is an exhausting sequence of compact sets in $\Omega$, where $\Omega \subseteq \mathbb{C}$ is an open set, and

$$
K_{r}=(\mathbb{C} \backslash \Omega) \cap\{z \in \mathbb{C}|| z \mid \leq r\}, \quad r \in \mathbb{N}
$$

Then, as in Application 4.1, $\left(L_{n}\right)_{n \in \mathbb{N}}$ defines the space $T^{\infty}\left(\Omega, L_{n}\right)=T^{\infty}(\Omega)$ which, as a set, equals $H(\Omega)$ and furthermore, it holds $Y^{\infty}(\Omega)=T^{\infty}(\Omega)=H(\Omega)$. Under these hypothesis Theorem 3.2 yields Theorem 3.4 of [13], according to which if $F$ is a subset of $\mathbb{N} \times \mathbb{N}$ that contains a sequence $\left(\widetilde{p}_{n}, \widetilde{q}_{n}\right), n=1,2, \ldots$ with $\widetilde{p}_{n} \rightarrow+\infty, q_{n} \rightarrow+\infty$, then generically every function $f \in H(\Omega)$ has universal Padé approximants obtained from the set $\{[f ; p / q] \mid(p, q) \in F\}$ that can approximate uniformly any rational function on any compact subset of $\mathbb{C} \backslash \Omega$ with respect to the chordal distance and can, simultaneously, approximate uniformly $f$ on any compact subset of $\Omega$ with respect to the Euclidean distance. One can see that in this case the universal approximation is valid on $\partial \Omega$.

4.3. We consider now the case where $\Omega \subseteq \mathbb{C}$ is a simply connected domain with a locally finite number of components, $\left(L_{n}\right)_{n \in \mathbb{N}}$ is an exhausting family of compact sets in $\Omega$ (Proposition 2.7) and $\left(K_{r}\right)_{r \in \mathbb{N}}$ is a sequence of compact sets in $\mathbb{C} \backslash \Omega$ with con- 
nected complement as in Proposition 2.3. The sequence $\left(L_{n}\right)_{n \in \mathbb{N}}$, as in Application 4.1, defined the space $T^{\infty}(\Omega)=T^{\infty}\left(\Omega,\left(L_{n}\right)_{n \in \mathbb{N}}\right)$ and it holds $T^{\infty}(\Omega)=Y^{\infty}(\Omega)=H(\Omega)$. Now, under these hypothesis, if the set $F \subset \mathbb{N} \times \mathbb{N}$ contains a sequence $\left(\widetilde{p}_{n}, \widetilde{q}_{n}\right)$ with $\widetilde{p}_{n} \rightarrow+\infty$, then Theorem 3.4 combined with Remark 3.5yields Theorem 3.4 of [5], according to which generically every holomorphic function $f \in H(\Omega)$ has universal Padé approximants obtained from the set $\{[f ; p / q] \mid(p, q) \in F\}$ that can approximate any polynomial function on any compact subset of $\mathbb{C} \backslash \Omega$ with connected complement with respect to the Euclidean distance and can, simultaneously, approximate $f$ with respect to the Euclidean distance on every compact subset of $\Omega$. We see that the universal approximation is also in this case valid on $\partial \Omega$. 4.4. We consider now the case where $L_{n}=\bar{\Omega} \cap \overline{D(0, n)}$ for every $n \in \mathbb{N}$ and $\left(K_{r}\right)_{r \in \mathbb{N}}$ is an exhausting sequence of compact sets in $\mathbb{C} \backslash \bar{\Omega}$. The existence of the sequence $\left(K_{r}\right)_{r \in \mathbb{N}}$ is established from Proposition 2.7.

Now, it is easy to see that $\left(L_{n}\right)_{n \in \mathbb{N}}$ is an increasing sequence of compact subsets of $\bar{\Omega}$ such that it holds $\overline{\left(L_{n} \cap \Omega\right)}=L_{n}$, for every $n \in \mathbb{N}$ and for every compact set $J \subset \Omega$, there exists $n \in \mathbb{N}$ such that $J \subset \bar{\Omega} \cap \overline{D(0, n)}=L_{n}$. For the last condition, let $n \in \mathbb{N}$. We will show that every connected component of $\{\infty\} \cup\left(\mathbb{C} \backslash L_{n}\right)$ contains a point from $\{\infty\} \cup(\mathbb{C} \backslash \bar{\Omega})$. It holds that $\{\infty\} \cup\left(\mathbb{C} \backslash L_{n}\right)=\{\infty\} \cup(\mathbb{C} \backslash \bar{\Omega}) \cup$ $(\mathbb{C} \backslash \overline{D(0, n)})$, and if $C$ is a connected component of $\{\infty\} \cup\left(\mathbb{C} \backslash L_{n}\right)$ it holds either $C \subset\{\infty\} \cup(\mathbb{C} \backslash \bar{\Omega})$ or $C \cap(\mathbb{C} \backslash \overline{D(0, n)}) \neq \emptyset$. In the former case the component $C$ obviously contains a point from $\{\infty\} \cup(\mathbb{C} \backslash \bar{\Omega})$ and in the latter case $C$ must contain the whole connected set $\{\infty\} \cup(\mathbb{C} \backslash \overline{D(0, n)})$ which means that $\{\infty\} \in C$ which is also a point from $\{\infty\} \cup(\mathbb{C} \backslash \bar{\Omega})$. Therefore, the space $T^{\infty}(\Omega)=T^{\infty}\left(\Omega,\left(L_{n}\right)_{n \in \mathbb{N}}\right)$ can be defined and because $\bigcup_{n} L_{n}$ contains any compact subset of $\bar{\Omega}, T^{\infty}(\Omega)$ becomes equal to the space $A^{\infty}(\Omega)$, which is the space of the functions $f \in H(\Omega)$ such that for every $\ell=0,1, \ldots f^{(\ell)}$ extends continuously on $\bar{\Omega}$, and $Y^{\infty}(\Omega)$ becomes equal to $X^{\infty}(\Omega)$, which is the closure of all rational functions with poles off $\bar{\Omega}$. Under these hypothesis Theorem 3.2 yields Theorem 3.3 of [14, according to which if $F$ is a subset of $\mathbb{N} \times \mathbb{N}$ that contains a sequence $\left(\widetilde{p}_{n}, \widetilde{q}_{n}\right)$ where $\widetilde{p}_{n} \rightarrow+\infty, \widetilde{q}_{n} \rightarrow+\infty$ then generically every function $f \in X^{\infty}(\Omega) \subseteq A^{\infty}(\Omega)$ has universal Padé approximants obtained from the set $\{[f ; p / q] \mid(p, q) \in F\}$ that can approximate uniformly any rational function on any compact subset of $\mathbb{C} \backslash \bar{\Omega}$ with respect to the chordal metric, and can, simultaneously, 
approximate $f$ on every compact subset of $\bar{\Omega}$ with respect to the Euclidean distance.

We see that in this case, not only the approximation is not valid on the boundary $\partial \bar{\Omega}$ but on the compact subsets of $\partial \Omega$ the universal Padé approximants approximate the function that defines them.

4.5. Consider now the case where $\Omega \subseteq \mathbb{C}$ is an open set with a locally finite number of components such that $\{\infty\} \cup(\mathbb{C} \backslash \bar{\Omega})$ is connected, $L_{n}=\bar{\Omega} \cap \overline{D(0, n)}$ for every $n \in \mathbb{N}$ and $\left(K_{r}\right)_{r \in \mathbb{N}}$ is an exhausting sequence of compact subsets of $\mathbb{C} \backslash \bar{\Omega}$ with connected complement as in Proposition 2.4.

Then, as in Application 4.4, $T^{\infty}(\Omega)=T^{\infty}\left(\Omega,\left(L_{n}\right)_{n \in \mathbb{N}}\right)$ coincides with the space $A^{\infty}(\Omega)$ and $Y^{\infty}(\Omega)$ coincides with $X^{\infty}(\Omega)$, where both $A^{\infty}(\Omega)$ and $X^{\infty}(\Omega)$ were defined in Application 4.4. Then Theorem 3.3 under these hypothesis yields that if $F$ is a subset of $\mathbb{N} \times \mathbb{N}$ that contains a sequence $\left(\widetilde{p}_{n}, \widetilde{q}_{n}\right), n=1,2, \ldots$ with $\widetilde{p}_{n} \rightarrow+\infty$, then generically every function $f \in X^{\infty}(\Omega) \subseteq A^{\infty}(\Omega)$ has universal Padé approximants obtained from the set $\{[f ; p / q] \mid(p, q) \in F\}$ that can approximate uniformly every polynomial function on every compact subset of $\mathbb{C} \backslash \bar{\Omega}$ with connected complement with respect to the Euclidean metric and can simultaneously approximate $f$ on every compact subset of $(\bar{\Omega})$. The above result is similar to the Theorem 4.1 of [14].

One can notice that also in this case the universal approximation is not valid on $\partial \Omega$.

bf 4.6. Now, we will present an example where on a part of the boundary $\partial \Omega$ the universal Padé approximation will be valid and on another part it will be not.

$$
\begin{gathered}
\text { Let } \Omega=D=\{z \in \mathbb{C}|| z \mid<1\} \text {, and for every } n \in \mathbb{N} \text { we set } \\
L_{n}=\{z \in \mathbb{C}|| z \mid \leq 1-1 / n\} \cup\{z \in \mathbb{C} \backslash\{0\}|| z \mid \leq 1,1 / n \leq \operatorname{Arg} z \leq \pi-1 / n\} .
\end{gathered}
$$

We also consider $\left(K_{r}\right) r=1, \ldots$ a sequence of compact subsets of $\mathbb{C} \backslash \bar{D}$ as in Proposition 2.4, and

$$
K_{1}=\{z \in \mathbb{C}|| z \mid \geq 1,-2 \leq \operatorname{Re}(z) \leq 2, \operatorname{Im}(z) \leq 0\} .
$$

It is easy to check that with this choice of $\left(L_{n}\right)_{n \in \mathbb{N}}$ the space $T^{\infty}(\Omega)=T^{\infty}\left(\Omega,\left(L_{n}\right)_{n \in \mathbb{N}}\right)$ is well defined and that for this space Theorem 3.4 yields the following theorem.

Theorem 4.1. Let $F$ be a subset of $\mathbb{N} \times \mathbb{N}$ such that $F \supset\left\{\left(\widetilde{p}_{n}, \widetilde{q}_{n}\right), n \in \mathbb{N}\right.$ with $\left.\widetilde{p}_{n} \rightarrow+\infty\right\}$ and let $\left(L_{n}\right)_{n \in \mathbb{N}}$ and $\left(K_{r}\right)_{r \in \mathbb{N}}$ be defined as above. Then, there exist a 
function $f \in H(D)$ such that for every polynomial $p$ and $r \in \mathbb{N}$ there exists a sequence $\left(p_{n}, q_{n}\right) \in F, n=1,2, \ldots$ such that for every member of the sequence $\left(L_{n}\right)_{n \in \mathbb{N}}, L_{N}$ there exists a natural number $m=m(N) \in \mathbb{N}$ such that:

(i) $f \in D_{p_{n}, q_{n}}(\zeta) \cap E_{p_{n}, q_{n}, \zeta}\left(K_{r} \cup L_{N}\right)$ for every $n \geq m$.

(ii) $\sup _{\zeta \in L_{N}} \sup _{z \in K_{r}}\left|\left[f ; p_{n} / q_{n}\right]_{\zeta}(z)-p(z)\right| \rightarrow 0$, as $n \rightarrow+\infty$.

(iii) For every $\ell \in \mathbb{N}_{0}$, $\sup _{\zeta \in L_{N}} \sup _{z \in L_{N}}\left|\left[f ; p_{n} / q_{n}\right]_{\zeta}^{(\ell)}(z)-f^{(\ell)}(z)\right| \rightarrow 0$, as $n \rightarrow+\infty$.

One can see that Theorem 4.1 in case $F=\{(n, 0) \mid n \in \mathbb{N}\}$ coincides with Theorem 3.1 of 8 .

Now, Theorem 4.1 for $r=1$, gives the existence of a function $f \in H(D)$ with Padé approximant $[f ; p / q],(p, q) \in F$, where $F \subset \mathbb{N} \times \mathbb{N}$ was defined above, that can approximate any polynomial function uniformly on any compact subset of $\left\{e^{i \vartheta} \mid 0<\vartheta<\pi\right\}$ and can, simultaneously, approximate $f$ uniformly on any compact subset of $\left\{e^{i \vartheta} \mid \pi \leq \vartheta<2 \pi\right\} \cup\{1\}$.

Therefore indeed we prove the existence of a function where the universal Padé approximation is valid on a part of the boundary $\partial \Omega$, for $\Omega=D$, and it is not valid on another part of it.

\section{$5 \quad$ A sufficient condition}

Considering Theorem 4.1 one can naturally ask whether one can find necessary and sufficient conditions about the subsets $S, T$ of the boundary $\partial \Omega$, of an open set $\Omega$, so that some Padé approximants of a holomorphic function $f \in H(\Omega)$ approximate universally any rational function on any compact subset of $(\mathbb{C} \backslash \bar{\Omega}) \cup T$ with respect to the chordal distance and can, simultaneously, approximate $f$ on any compact subset of $\Omega \cup S$ with respect to the Euclidean distance. For convenience, we denote the set of such functions by $V(S, T, \Omega)$, possibly empty.

A necessary condition for $V(S, T, \Omega) \neq \emptyset$ is of course $S \cap T=\emptyset$. We will prove now that a sufficient condition for $S, T \subseteq \partial \Omega$ so that $V(S, T, \Omega) \neq \emptyset$ is $\bar{S} \cap \bar{T}=\emptyset$; i.e. $S, T$ have disjoint closures.

Proposition 5.1. Let $\Omega \subseteq \mathbb{C}$ be an open set and $S, T \subseteq \partial \Omega$ such that $\bar{S} \cap \bar{T}=\emptyset$. Then $V(S, T, \Omega) \neq \emptyset$. 
Proof. Based on Theorem 3.2 , it is enough to find two sequences $\left(L_{n}\right)_{n \in \mathbb{N}},\left(K_{r}\right)_{r \in \mathbb{N}}$ of compact sets in $\bar{\Omega}$ and in $\mathbb{C} \backslash \Omega$, respectively, such that: Firstly, for every $r, n \in \mathbb{N}$ it holds $K_{r} \cap L_{n}=\emptyset$, secondly $\left(L_{n}\right)_{n \in \mathbb{N}}$ fulfills the prerequisites to define the space $T^{\infty}(\Omega)=T^{\infty}\left(\Omega,\left(L_{n}\right)_{n \in \mathbb{N}}\right)$ and finally for any choice of compact subsets $J_{1} \subset \Omega \cup S$, $J_{2} \subset(\mathbb{C} \backslash \bar{\Omega}) \cup T$ there exist $R, N \in \mathbb{N}$ such that $J_{1} \subset L_{N}$ and $J_{2} \subset K_{R}$.

Because $\bar{S}, \bar{T}$ are closed, and therefore locally compact subsets of $\partial \Omega$, from Proposition 2.7 there exists an exhausting sequence of compact sets in $\bar{S}$, call it $\left(L_{n}^{\prime}\right)_{n \in \mathbb{N}}$ and an exhausting sequence of compact sets in $\bar{T}$, call it $\left(K_{r}^{\prime}\right)_{r \in \mathbb{N}}$. Now, as $\bar{S} \cap \bar{T}=\emptyset$, for every $n \in \mathbb{N}$ there exists a positive number $a_{n}>0$, such that $2 a_{n}<\min \left(\operatorname{dist}\left(L_{n}^{\prime} \bar{T}\right)\right.$, $\left.\operatorname{dist}\left(\bar{S}, K_{n}^{\prime}\right)\right)$.

Let also $\left(L_{n}^{\prime \prime}\right)_{n \in \mathbb{N}},\left(K_{r}^{\prime \prime}\right)_{r \in \mathbb{N}}$ be two exhausting sequences of compact sets in $\Omega$ and $\mathbb{C} \backslash \bar{\Omega}$ respectively; the existence of them is established from the fact that they are open subsets of $\mathbb{C}$ combined with Proposition 2.7. Now, we set for every $n \in \mathbb{N}$,

$$
\left.\begin{array}{l}
L_{n}=\left(\bigcup_{N=1}^{n}\left\{z \in \bar{\Omega} \mid \operatorname{dist}\left(z, L_{N}^{\prime}\right) \leq a_{N}\right\}\right) \cup L_{n}^{\prime \prime} \\
\text { and for every } r \in \mathbb{N}, \\
K_{r}=\left(\bigcup_{R=1}^{r}\left\{z \in \bar{\Omega} \mid \operatorname{dist}\left(z, K_{R}^{\prime}\right) \leq a_{R}\right) \cup K_{r}^{\prime \prime} .\right.
\end{array}\right\} \text { Definitions }(*)
$$

We first prove that for every $r, n \in \mathbb{N}, K_{r} \cap L_{n}=\emptyset$. Indeed, let $z \in L_{n} \cap K_{r}$. Then obviously $z \in \partial \Omega$ and as $L_{n}^{\prime \prime} \subset \Omega$ and $K_{r}^{\prime \prime} \subset \mathbb{C} \backslash \bar{\Omega}$ we get that there exist $N \in\{1,2, \ldots, n\}$ and $R \in\{1,2, \ldots, r\}$ such that

$$
z \in\left\{z \in \bar{\Omega} \mid \operatorname{dist}\left(z, L_{N}^{\prime}\right) \leq a_{N}, \operatorname{dist}\left(z, K_{R}^{\prime}\right) \leq a_{R}\right\}
$$

This means that there exist $\ell_{N} \in L_{N}, k_{R} \in K_{R}$ such that $\left|z-\ell_{N}\right| \leq a_{N},\left|z-k_{R}\right| \leq a_{R}$ which gives $\left|\ell_{N}-k_{R}\right| \leq a_{R}+a_{N}$ and therefore $\left|\ell_{N}-k_{R}\right|<2 \max \left(a_{R}, a_{N}\right)$.

Without loss of generality, we may assume $a_{N}>a_{R}$ and therefore that it holds $\left|\ell_{N}-k_{R}\right| \leq 2 a_{N}$. The above relation yields $\operatorname{dist}\left(L_{N}^{\prime}, \bar{T}\right) \leq \operatorname{dist}\left(L_{N}^{\prime}, K_{R}^{\prime}\right) \leq 2 a_{N}$, which is a contradiction. Therefore, indeed for every $n, r \in \mathbb{N}, L_{n} \cap K_{r}=\emptyset$.

Now, it is easy to see that $\left(L_{n}\right)_{n \in \mathbb{N}}$ is an increasing sequence of compact subsets of $\bar{\Omega}$, that for every $n \in \mathbb{N}$ it holds $\overline{\left(L_{n} \cap \Omega\right)}=L_{n}$, and that for every compact subset of $\Omega, J \subset \Omega$, there exists $N \in \mathbb{N}$ such that $J \subset L_{N}^{\prime \prime} \subset L_{N}$.

These three facts and Corollary 2.9 show that $\left(L_{n}\right)_{n \in \mathbb{N}}$ can be modified such that for every $n \in \mathbb{N}$ every connected component of $\{\infty\} \cup\left(\mathbb{C} \backslash L_{n}\right)$ will contain a point from $\{\infty\} \cup(\mathbb{C} \backslash \bar{\Omega})$, leaving however, for every $n \in \mathbb{N}$ the set $L_{n} \cap \partial \Omega$ unchanged. 
One can check that the new sequence $\left(L_{n}\right)_{n \in \mathbb{N}}$ fulfills all the prerequisites to define the space $T^{\infty}(\Omega)=T^{\infty}\left(\Omega,\left(L_{n}\right)_{n \in \mathbb{N}}\right)$ and that it remains true that $L_{n} \cap K_{r}=\emptyset$, for every $r, n \in \mathbb{N}$. The former is immediate. For the latter; let $r, n \in \mathbb{N}$. Then, as $L_{n} \subset \bar{\Omega}, K_{r} \subset \mathbb{C} \backslash \Omega$ it must also be true that $L_{n} \cap K_{r} \subset \partial \Omega$ and therefore $L_{n} \cap K_{r}=$ $\left(L_{n} \cap \partial \Omega\right) \cap\left(K_{r} \cap \partial \Omega\right)$. The last equation together with the fact that $\left(L_{n} \cap \partial \Omega\right)$ remained unchanged through the modification yields that indeed the intersection $L_{n} \cap K_{r}$ also remains equal to the empty set. Finally, we have to show that for any choice of compact subsets $J_{1} \subset \Omega \cup S$ and $J_{2} \subset(\mathbb{C} \backslash \bar{\Omega}) \cup T$, there exist $R, N \in \mathbb{N}$ such that $J_{1} \subset L_{N}$, $J_{2} \subset K_{R}$. As the new sequence $\left(L_{n}\right)_{n \in \mathbb{N}}$ contains the old one, we may use for $\left(L_{n}\right)_{n \in \mathbb{N}}$ the one we defined at $(*)$.

Now, because of the symmetry of the definitions $(*)$, it is enough to prove that for every compact set $J_{1} \subset \Omega \cup S$, one can find $N \in \mathbb{N}$ such that $J_{1} \subset L_{N}$. Indeed, let $J_{1}$ be a compact subset of $\Omega \cup S$. One can see that because $J_{1} \cap S$ is a compact subset of $\partial \Omega$ and $\left(L_{n}^{\prime}\right)_{n \in \mathbb{N}}$ is an exhausting sequence of compact sets in $\partial \Omega$, there exists $n_{1} \in \mathbb{N}$ such that $J_{1} \cap S \subset L_{n_{1}}$. (Proposition 2.6).

Now, we claim that there exists $M \in \mathbb{N}$ such that

$$
J_{1} \cap\{z \in \bar{\Omega} \mid \operatorname{dist}(z, \partial \Omega)<1 / M\} \subseteq\left\{z \in \bar{\Omega} \mid \operatorname{dist}\left(z, L_{n_{1}}^{\prime}\right) \leq a_{n_{1}}\right\} .
$$

Indeed, if not there exists a sequence $\left(x_{n}\right)_{n \in \mathbb{N}} \subset J_{1}$ such that $\operatorname{dist}\left(x_{n}, \partial \Omega\right) \rightarrow 0$ and $\operatorname{dist}\left(x_{n}, L_{n_{1}}^{\prime}\right) \geq a_{n_{1}}$, for every $n \in \mathbb{N}$. These two relations together with the fact that $J_{1}$ is compact yield a point $z_{0} \in J \cap \partial \Omega$ with $\operatorname{dist}\left(z_{0}, L_{n_{1}}^{\prime}\right) \geq a_{n_{1}}$. But this is a contradiction since $J_{1} \cap \partial \Omega=J_{1} \cap S \subset L_{n_{1}}^{\prime}$.

Now, fix such a number $M \in \mathbb{N}$. We have that $J_{1} \cap\{z \in \bar{\Omega} \mid \operatorname{dist}(z, \partial \Omega)<1 / M\} \subset$ $L_{n_{1}}$, by definitions of $M, L_{n_{1}}$. Moreover, since $J_{1} \cap\{z \in \bar{\Omega} \mid \operatorname{dist}(z, \partial \Omega) \geq 1 / M\}$ is a compact subset of $\Omega$, there exists $n_{2} \in \mathbb{N}$ such that

$$
J_{1} \cap\{z \in \bar{\Omega} \mid \operatorname{dist}(z, \partial \Omega) \geq 1 / M\} \subset L_{n_{2}}^{\prime \prime} \subset L_{n_{2}},
$$

because $\left(L_{n}^{\prime \prime}\right)_{n \in \mathbb{N}}$ is an exhausting sequence of compact sets in $\Omega$.

Therefore, it holds:

$$
\begin{aligned}
J_{1}= & \left(J _ { 1 } \cap \{ z \in \overline { \Omega } | \operatorname { d i s t } ( z , \partial \Omega ) < 1 / M ) \cup \left(J_{1} \cap\{z \in \bar{\Omega} \mid \operatorname{dist}(z, \partial \Omega) \geq 1 / M)\right.\right. \\
& \subseteq L_{n_{1}} \cup L_{n_{2}} \subset L_{n_{1}+n_{2}}=L_{N},
\end{aligned}
$$

for $N=n_{1}+n_{2} \in \mathbb{N}$, which is what we wanted. The proof of Proposition 5.1 is complete. 
Remark 5.2. In the case where $\Omega \subseteq \mathbb{C}$ is a bounded open set, then one can simplify the above proof of Proposition [5.1, be replacing each $L_{n}^{\prime}$ by $\bar{S}$ and each $K_{r}^{\prime}$ by $\bar{T}$.

Corollary 5.3. Let $\Omega$ be an open set and $S, T \subseteq \mathbb{C}$ be two closed subsets of $\partial \Omega$. Then, it holds $V(S, T, \Omega) \neq \emptyset$ if and only if $S \cap T=\emptyset$.

Corollary 5.4. Let $\Omega$ be an open set. Then, if $S \subseteq \partial \Omega$ is a closed set, there exists a function $f \in H(\Omega)$, such that for every $\ell \in \mathbb{N}$, the derivative $f^{(\ell)}$ extends continuously on $\Omega \cup S$.

The total characterization of the subsets $S, T$ of the boundary $\partial \Omega$ of an open set $\Omega$ such that $V(S, T, \Omega) \neq \emptyset$ remains an open problem.

Acknowledgement. I would like to thank Professor V. Nestoridis for many helpful discussions and guidance through the creation of this paper.

\section{References}

[1] G. A. Baker, Jr and P. R. Graves-Morris, Padé Approximants. Bol. 1 and 2 (Encyclopedia of Math. and Applications), Cambridge, Un. Press 2010.

[2] Bayart, Grosse-Erdmann, Nestoridis and Papadimitropoulos, Abstract theory of Universal series and applications, Proceedings of the Lon Math. Soc. (3) 96(2008) no $2,417-463$.

[3] C. Chui and M. N. Parnes, Approximation by overconvergence of power series, J. Math. Anal. Appl. 36(1971), 693-696.

[4] N. Daras, V. Nestoridis and Ch. Papadimitropoulos, Universal Padé approximation of Selezner type, Arch. Math. (Basel), 100(2013) no 6 571-585.

[5] N. Daras, G. Fournodavlos, V. Nestoridis, Universal Padé approximants on simply connected domains, submitted.

[6] G. Fournodavlos, V. Nestoridis, Generic approximation of functioning by their Padé approximants, Journal of Mathematical Analysis and Application, vol. 408, issue 2 (2013), 744-750.

[7] Ch. Kariofillis, Ch. Konstadilaki and V. Nestoridis, Smooth universal Taylor Series, Monasth. Math. 147(2006) no. 3, 249-257. 
[8] Ch. Kariofillis, V. Nestoridis: Universal Taylor series in Simly Connected Domains, C.M.F.T., Volume 6 (2006), No. 2, 437-446.

[9] W. Luh (1970), Approximation analytisher Funktionen dürch überkonvergente Potenzreihen und dere Matrix-Transformierten. Mitt Math Sem Giessen 88:1-56.

[10] A. Melas and V. Nestoridis: Universality of Taylor series as a generic property of holomorphic functions, Adv. Math. Vol. 157, 2001, p. 138-176.

[11] V. Nestoridis, Universal Taylor series, Ann. Inst. Fourier 46 5(1996), 1293-1306.

[12] V. Nestoridis, An extension of the notion of universal Taylor series in: N. Papamichael, S. Ruscheweyh, E. B. Saff (eds), Proceedings of the 3rd CMFT conference on computational methods and function theory 1997, Nicosia, Cyprus, October 13-17, 1997, World Scientific. Ser. Approx. Decompos. 11(1999), 421-430.

[13] V. Nestoridis, Universal Padé Approximants with respect to the chordal metric, Journal of Contemporary Mathematics Analysis, v. 47(2012) no 4, 168-181.

[14] V. Nestoridis, I. Zadik, Padé approximants, density of rational functions in $A^{\infty}(\Omega)$ and smoothness of the integration operator, arXiv:1212.4394.

[15] J. Pál (1914-1915) Zwei Kleine Bemerkungen, Tôhoku Math J 6: 42-43.

University of Athens

Department of Mathematics

15784 Panepistimiopolis

Athens

GREECE

email address:ilias__91@hotmail.com 